\title{
COMPARISON OF A QUALITATIVE CANINE C-REACTIVE PROTEIN TEST TO A QUANTITATIVE TEST AND TRADITIONAL MARKERS OF INFLAMMATION - SHORT COMMUNICATION
}

\author{
Celia M. SMUTS* , Jennifer N. MiLls and Tibor GAÁL \\ Department of Veterinary Clinical Pathology, Murdoch University, 90 South Street, \\ Murdoch, 6150 Western Australia, Australia
}

(Received 7 March 2015; accepted 22 June 2015)

\begin{abstract}
Qualitative tests for C-reactive protein (CRP) are available for use in dogs, and provide a rapid in-house method of detecting acute inflammation. The aim of this study was to compare results from a qualitative CRP lateral flow test (Teco CRP FASTest) to those obtained from a quantitative CRP ELISA and to traditional methods of detecting inflammation, including total leukocyte and neutrophil numbers, presence of immature neutrophils and a left shift, presence or absence of toxic changes in neutrophils and plasma fibrinogen concentration in whole blood and serum samples collected from 113 client-owned dogs. More dogs had CRP FASTest positive results than had quantitatively increased CRP (ELISA) or increases in traditional methods used for measuring inflammation. Few dogs had increases in markers of inflammation but no elevated CRP. The qualitative CRP FASTest was found to be a sensitive test for detecting increased CRP concentration and was positive more frequently than were traditional markers of inflammation.
\end{abstract}

Key words: Canine, C-reactive protein, point-of-care laboratory test

Making an early diagnosis of acute inflammation is important for clinicians both in human and veterinary medicine. Detection of non-specific clinical signs of inflammation (e.g. fever, inappetence, lethargy) is complemented by laboratory methods that have been developed for detecting inflammation. Traditionally the presence of leukocytosis and neutrophilia, left shifts and toxic changes in neutrophils, elevated plasma fibrinogen concentrations and accelerated erythrocyte sedimentation rate have been widely used in veterinary laboratories to assess acute inflammatory processes (Jain, 1986). These traditional methods have gradually been replaced or supplemented with measurement of acute phase proteins (APPs), firstly in human, then in veterinary laboratory medicine (Eckersall and Conner, 1988; Kjelgaard-Hansen and Jacobsen, 2011).

*Corresponding author; E-mail: c.smuts@murdoch.edu.au, Phone: 0061 (8) 9360-7429 
C-reactive protein (CRP) is considered one of the major positive APPs in dogs due to the magnitude of the response in inflammatory processes (Ceron et al., 2005; Tecles et al., 2005). Canine CRP has been isolated and characterised (Caspi et al., 1984; Yamamoto et al., 1992; Jasensky et al., 2014). It is found physiologically in very low serum concentrations in the blood of animals without inflammatory disease and a rapid increase in CRP is part of an inflammatory response (Ceron et al., 2005), concentration rising as soon as 4 hours after an inflammatory stimulus (Caspi et al., 1984). CRP binds directly to dying cells and some bacteria, acting as an opsonin, and activates the complement system by the classical C1q pathway (Petersen et al., 2004; Jain et al., 2011).

Unfortunately, quantitative measurement of APP concentrations using an ELISA or fluorometric assay is possible only in well-equipped laboratories. A relatively new assay, TECOmedical Dog CRP-visual ${ }^{\circledR}$ (Sissach, Switzerland), a qualitative canine-specific lateral flow POC assay, showed good agreement with a quantitative CRP ELISA method used under laboratory conditions (Plickert et al., 2011).

The aim of this study was to compare the use of the qualitative TECOmedical Dog CRP-visual FASTest with the quantitative TECO ${ }^{\circledR}$ Canine CRP ELISA, as well as with other more traditional tests used to evaluate inflammation (presence of leukocytosis, neutrophilia, left shift and toxic changes in neutrophils, and determination of fibrinogen concentration measured by heat precipitation in blood plasma). Left shift and toxic changes were included to help in distinguishing between stress-induced (mature neutrophilia) and inflammatory leukograms. The qualitative CRP test has not, to the authors' knowledge, been compared to traditional laboratory methods used for detecting inflammation.

Canine blood samples that were submitted in both EDTA and plain (clotted) tubes to the Clinical Pathology Laboratory of Murdoch University (113 client-owned dogs) over a 6-week period underwent a complete blood count as well as CRP detection measured using both the Teco CRP point-of-care (POC) lateral flow FASTest and the Teco CRP quantitative ELISA. The samples had been collected for diagnostic purposes and animal ethics approval was not required for further testing.

Haematological analysis of each EDTA sample was performed using the Siemens Advia 120 haematology system (Bayer, Germany). Leukocytosis was recorded if the white cell count exceeded $13.9 \times 10^{9} / \mathrm{L}$ and neutrophilia if neutrophil numbers were $>8.0 \times 10^{9} / \mathrm{L}$. Neutrophils were evaluated on a WrightGiemsa (Bayer, Germany) stained blood smear for the presence or absence of toxic changes (Döhle bodies, cytoplasmic basophilia) and left shift (immature neutrophils $>0.3 \times 10^{9} / \mathrm{L}$ ) by a technician and clinical pathologist.

Fibrinogen concentration was measured in 95 EDTA samples using the heat precipitation method (Jain, 1986). The total solids of plasma (TSP) of the supernatant from each capillary tube was measured using a temperature- 
calibrated refractometer (Reichert Vet 360, NY, USA) and the TSP from the heated tube subtracted from the TSP of the unheated tube to calculate fibrinogen concentration. Samples with values $>4 \mathrm{~g} / \mathrm{L}$ were classified as inflammatory.

Samples collected in plain (serum) tubes were centrifuged and the serum was retained. C-reactive protein was measured in the serum within $2 \mathrm{~h}$ of sample collection using the qualitative TECO FASTest (Megacor Diagnostik, Germany) performed according to the manufacturer's instructions. The serum samples were then frozen at $-20{ }^{\circ} \mathrm{C}$ and stored until all the samples had been collected (approximately 2 months). Previous studies have shown that CRP is relatively stable even after repeated freeze-thaw cycles (Aziz et al., 2003). CRP was quantified in the serum samples using the Teco CRP ELISA ${ }^{\circledR}$ (Teco Medical Group, Switzerland) according to the manufacturer's instructions. Optical density was measured at $450 \mathrm{~nm}$ using an iMark Microplate Absorbance Reader (BioRad Laboratories, USA). The manufacturer suggests that sample values of $\geq 5 \mathrm{mg} / \mathrm{L}$ are positive for CRP stimulation associated with acute inflammation and samples with $>5 \mathrm{mg} / \mathrm{L}$ were recorded as inflammatory.

Data were analysed using Excel $^{\circledR}$ (Microsoft Office Enterprise, 2007, USA) to determine median values and standard error, and Spearman's rank correlation was used to compare results from different tests. Unpaired $t$-tests were used to compare results from the CRP ELISA and traditional markers of inflammation between CRP FASTest positive and negative samples.

The samples were collected from a variety of canine breeds and crossbreeds, entire and neutered males and females. The ages of the dogs ranged from 17 weeks to 14 years. Samples were included from dogs presented for preoperative screening and potential blood donors as well as from those with clinically suspected inflammatory diseases.

Of the 113 samples the lateral flow POC test detected 57 samples positive and 56 samples negative for CRP. The samples that were negative with the CRP FASTest gave quantitative ELISA CRP values that ranged from 2.05 to $4.64 \mathrm{mg} / \mathrm{L}$, apart from one sample that gave an ELISA result of $48.85 \mathrm{mg} / \mathrm{L}$. The results are summarised in Table 1.

\section{Table 1}

Range and mean values from all samples tested by CRP FASTest for CRP ELISA and traditional markers of inflammation

\begin{tabular}{lccccc}
\hline & $\begin{array}{c}\text { CRP ELISA } \\
\mathrm{mg} / \mathrm{L}\end{array}$ & $\begin{array}{c}\text { WBC } \\
\left(\times 10^{9} / \mathrm{L}\right)\end{array}$ & $\begin{array}{c}\text { Neutrophils } \\
\left(\times 10^{9} / \mathrm{L}\right)\end{array}$ & $\begin{array}{c}\text { Band neutrophils } \\
\left(\times 10^{9} / \mathrm{L}\right)\end{array}$ & $\begin{array}{c}\text { Fibrinogen } \\
(\mathrm{g} / \mathrm{L})\end{array}$ \\
\hline Range & $2.05-54.8$ & $2.6-84.0$ & $0.6-44.9$ & $0-17.6$ & $0-6$ \\
Mean of negatives & 3.9 & 10.1 & 7.2 & 0.19 & 2 \\
Mean of positives & 30.5 & 15.3 & 12.4 & 1.7 & 3
\end{tabular}

Characterisation in positives and negatives based on the CRP FASTest 
There was a significant increase $(\mathrm{P}<0.001)$ in CRP concentration measured by ELISA, total leukocyte count, neutrophil numbers and left shift, toxic changes and fibrinogen concentration in CRP FASTest positive samples compared to those that were CRP FASTest negative (Fig. 1).

\section{CRP ELISA}

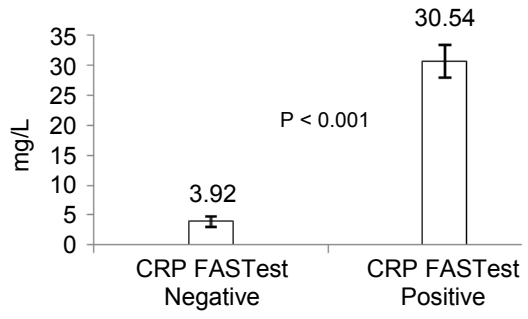

Total neutrophil count

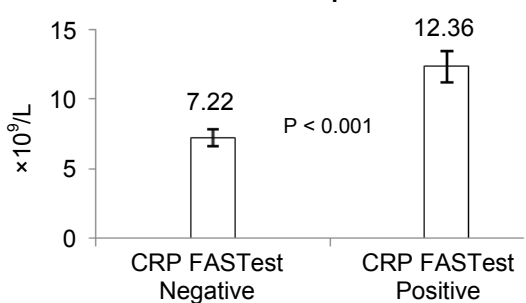

Toxic changes

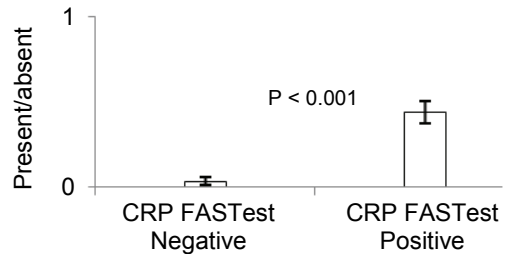

Total leukocyte (WBC) count

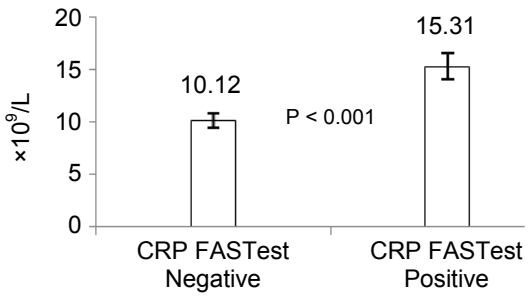

Band neutrophils

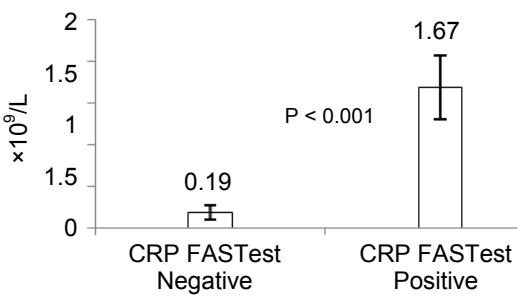

Fibrinogen

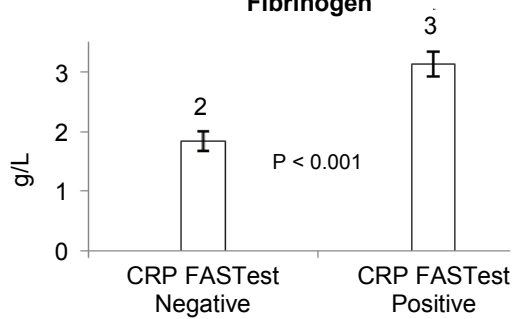

Fig. 1. Histograms comparing the mean \pm standard error (SE) of quantitative CRP concentration, total leukocyte count, neutrophil count, neutrophil left shift, presence of toxic changes and fibrinogen concentration between samples which tested negative versus those that tested positive for CRP using the CRP FASTest

There was good correlation between the CRP ELISA and FASTest $(r=$ 0.83 ) but only moderate to poor correlation between the CRP FASTest and total leukocyte count $(r=0.43)$, total neutrophil count $(r=0.38)$, immature neutrophil numbers $(r=0.33)$, fibrinogen concentration $(r=0.47)$ and presence or absence of toxic changes $(\mathrm{r}=0.34)$. 
Six of the samples with high total leukocyte counts $\left(15.7-84.0 \times 10^{9} / \mathrm{L}\right)$ gave negative test results using both the CRP lateral flow FASTest and CRP ELISA. The clinical history showed that all 6 dogs were receiving immunosuppressive therapy.

The CRP FASTest appears to be a reliable screening test for CRP as all the samples with quantitative ELISA CRP concentrations higher than $5 \mathrm{mg} / \mathrm{L}$ gave positive CRP FASTest results. This supports the conclusions of another study (Plickert et al., 2011). The CRP FASTest was positive in 9 samples (8\%) with negative CRP ELISA results. This may be due to the higher sensitivity of the FASTest or increased false positives detected by the CRP FASTest.

The CRP FASTest was more frequently positive in dogs with quantitatively increased CRP concentration than other commonly used laboratory methods used for detecting inflammation including leukocytosis, neutrophilia, left shift, toxic changes and plasma fibrinogen concentration; however, these parameters were significantly increased in the CRP FASTest positive group compared to the CRP FASTest negative group. The increases in traditional makers of inflammation with negative CRP FASTest results may be due to a more chronic inflammatory process, and acute phase proteins may therefore already have decreased. Further investigations looking at leukocyte count and morphology over time would be of value.

A shortcoming of our study is that only limited numbers of samples from animals treated with immunosuppressant drugs were tested. Previous studies have suggested that corticosteroids may not influence CRP concentration in healthy dogs (Martinez-Subiela et al., 2004) and may lower CRP concentration in dogs with sepsis (Caldin et al., 2009). Other explanations for a low CRP concentration with leukocytosis may include chronic inflammation where the CRP has already decreased, or a physiological leukocytosis without inflammation.

Other limitations of this study included the lack of follow-up samples to compare progression of inflammatory changes compared to CRP concentration. Sampling to follow the progression of disease would help to determine whether traditional indicators of inflammation occurred after CRP concentration increased, or vice versa. An advantage of a qualitative CRP test over a quantitative ELISA test is the production of a rapid result and easy means of testing. A major disadvantage of this simple-to-do CRP FASTest is that it only indicates the presence or absence of elevated CRP. Therefore this test cannot be used to evaluate the progression of disease or variations in inflammatory response.

In conclusion, the CRP FASTest ${ }^{\circledR}$ is a quick, easy-to-use test that is sensitive for detection of elevated CRP and can be performed on individual canine blood samples in a practice setting. It is more sensitive at detecting increased CRP than more traditional markers of inflammation. 


\section{Acknowledgements}

Our thanks go to TECO Medical AG in Switzerland who provided both the CRP FASTest qualitative and CRP quantitative ELISA test for dogs. Thanks also to the staff of Murdoch University Clinical Pathology Laboratory for technical help.

\section{References}

Aziz, N., Fahey, J. L., Detels, R. and Butch, A. W. (2003): Analytical performance of a highly sensitive C-reactive protein-based immunoassay and the effects of laboratory variables on levels of protein in blood. Clin. Diagn. Lab. Immun. 10, 652-657.

Caldin, M., Tasca, S., Carli, E., Bianchini, S., Furlanello, T., Martinez-Subiela, S. and Ceron, J. J. (2009): Serum acute phase protein concentrations in dogs with hyperadrenocorticism with and without concurrent inflammatory conditions. Vet. Clin. Path. 38, 63-68.

Caspi, D., Baltz, M. L., Snel, F., Gruys, E., Niv, D., Batt, R. M., Munn, E. A., Buttress, N. and Pepys, M. B. (1984): Isolation and characterization of C-reactive protein from the dog. Immunology 53, 307-313.

Ceron, J. J., Eckersall, P. D. and Martinez-Subiela, S. (2005): Acute phase proteins in dogs and cats: current knowledge and future perspectives. Vet. Clin. Path. 34, 85-99.

Eckersall, P. D. and Conner, J. G. (1988): Bovine and canine acute phase proteins. Vet. Res. Commun. 12, 169-178.

Jain, N. C. (ed.) (1986): Schalm's Veterinary Hematology. Lea and Febiger, Philadelphia. pp. 161, 1096.

Jain, S., Gautam, V. and Naseem, S. (2011): Acute-phase proteins: as diagnostic tool. J. Pharm. Bioallied Sci. 3, 118-127.

Jasensky, A. K., Bondzio, A., Murugaiyan, J., Siebert, U., Roesler, U., Kohn, B. and Einspanier, R. (2014): Characterization of the native C-reactive protein (cCRP) and the corresponding liver mRNA in dogs. Biochem. Biophys. Res. Commun. 452, 462-467.

Kjelgaard-Hansen, M. and Jacobsen, S. (2011): Assay validation and diagnostic applications of major acute-phase protein testing in companion animals. Clin. Lab. Med. 31, 51-70.

Martinez-Subiela, S., Ginel, P. J. and Ceron, J. J. (2004): Effects of different glucocorticoid treatments on serum acute phase proteins in dogs. Vet. Rec. 154, 814-817.

Petersen, H. H., Nielsen, J. P. and Heegaard, P. M. H. (2004): Application of acute phase protein measurements in veterinary clinical chemistry. Vet. Res. 35, 163-187.

Plickert, H. D., Einspanier, R., Arndt, G., Brunnberg, L. and Kohn, B. (2011): Evaluation of a point-of-care test for canine C-reactive protein. Vet. Clin. Path. 40, 384-388.

Tecles, F., Spiranelli, E., Bonfanti, U., Ceron, J. J. and Paltrinieri, S. (2005): Preliminary studies of serum acute-phase protein concentrations in hematologic and neoplastic diseases of the dog. J. Vet. Intern. Med. 19, 865-870.

Yamamoto, S., Tagata, K., Nagahata, H., Ishikawa, Y., Morimatsu, M. and Naiki, M. (1992). Isolation of canine C-reactive protein and characterization of its properties. Vet. Immunol. Immunopathol. 30, 329-339. 